\title{
Teaching Sessions
}

\section{Dementia: Evidence-Based Medicine and Epidemiological Update}

\section{Amos D. Korczyn \\ Sieratzki Chair of Neurology, Tel-Aviv University Medical \\ School, Ramat Aviv, Israel}

Alzheimer's disease (AD), vascular dementias (VaD), Lewy body dementias, and frontotemporal dementia all have a complex presentation and challenges in the diagnostic criteria make none of these 'causes' truly correct. It is more instructive to see these entities on a continuum of neuropathological conditions.

By example, the DSM-IV criteria do not do justice to the variety of syndromes seen in AD. The specific wordings of the criteria leave much room for interpretation. These criteria require impairment of function; if memory impairment or another symptom is not severe enough to demonstrate functional impairment in activities of daily living, the diagnosis should not be dementia. Diagnostic criteria disregard mixed cases and are mostly useful for research criteria only, not epidemiological field work. Clearly, it is difficult regarding who to count.

Nevertheless, the non-genetic risk factors based on epidemiological studies showed that in AD, age is the single most important factor. The prevalence increases with age, at about two-fold for every 5 years in patients aged $>65$ years; this means by 85 years of age, the prevalence is $\sim 20 \%$, at 90 , at $\sim 40 \%$. Beyond 90 years, there is little data. When genetic considerations are taken into account, these data may be relevant to finding individuals who are "coded" to survive.

What happens to an individual when they are young can also affect their risk of dementia later in life. Head trauma with concomitant short-duration coma (3-4 days) seems to confer a small increased risk. This injury may cause a low-level effect that may remain active over decades; however, the underlying processes are not understood.

Another risk factor is a low education level. Several studies have demonstrated that higher education (versus no schooling) confers a protection of 10 to 1 , i.e. $90 \%$ of schooled individuals did not develop dementia compared to their unschooled agematched counterparts. Occupational attainment showed a similar protective effect, although whether this is due to higher education can not be ruled out. These data emphasize from a social level, the impact that non-genetic risk factors have from a young age on the future burden of dementia.

Younger individuals ( $<65$ years) with symptoms matching the DSM-IV criteria have a higher likelihood to have a strong genetic component. In early-onset $\mathrm{AD}$, autosomal dominant inheritance (3 generations affected, e.g. PSEN1) is possible, whereas in lateonset AD, APOE genetic testing may be useful; however, AD is genetically heterogeneous. The APOE $\varepsilon 4$ may indicate that the dementia is of vascular origin as it only advances the onset, it doesn't change the risk. For patients aged $>65$ years, genetic explanations are much less likely. Moreover, genetic analysis should not be considered deterministic; it simply offers the patient, family and doctor a chance to be more careful and proactive about prevention.

The risk factors of coronary artery disease, smoking, hyperhomocysteinemia, diabetes mellitus, hypertension, high dietary saturated fat and cholesterol are critical, particularly at mid-life. Studies demonstrated that the status at mid-life is still a risk factor, even if one quits smoking at later date. The cumulative effect of these vascular risk factors was shown elegantly by Kivipelto et al. [1]. These 'hyper' risk factors for atherosclerosis, stroke and $\mathrm{AD}$ share commonalities with dementia, even after correcting for vascular disease.

However, the poor definitions and lack of data make it difficult to distinguish between AD and VaD. The DSM-IV criteria are even more difficult to apply here: relying on memory impairments, deciding whether a cardiovascular lesion is due to cognitive impairment, stroke cases with prior physical incapacity versus depression; these are not adequate for the differential in clinical practice.

After death, MRI of the intact brain would be useful if it could be collected systematically. There are possibly many clinically silent lesions on MRI and, though it probably won't be clear-cut between $\mathrm{AD}$ and $\mathrm{VaD}$, the information could be more compelling than self-reporting and questionnaires in dementia. Neurochemical methods have thus far also not been able to provide a discriminating factor. Lastly, the pathology in dementia is not consistent; an excellent example of this is the Nun Study [2].

When the origins are so obviously different can one really classify all of these conditions and signs as the same disease? The clinical perspective should be that almost all dementia is mixed dementia. Experience shows that $\mathrm{VaD}$ may mimic $\mathrm{AD}$ course or there may be interactions between the 2 'causes' as seen on follow-up. As the history of the classifications is convoluted, why should we differentiate at all? Conceptually correct, however, in practice the separation is impractical and unproductive. It is important to remember that even if one predominates, this does not mean the other one is unimportant or not exerting an effect. In an ageing society, this burden of disease is at an unstable condition; preventing dementia in younger people through social means could be helpful long term.

\section{References}

1 Kivipelto M, Helkala EL, Laakso MP, Hänninen T, Hallikainen M, Alhainen K, Iivonen S, Mannermaa A, Tuomilehto J, Nissinen A, Soininen $\mathrm{H}$ : Apolipoprotein E epsilon4 allele, elevated midlife total cholesterol level, and high midlife systolic blood pressure are independent risk factors for late-life Alzheimer disease. Ann Intern Med 2002;137:149-155.

2 Snowdon DA, Greiner LH, Mortimer JA, Riley KP, Greiner PA, Markesbery WR: Brain infarction and the clinical expression of Alzheimer disease. The Nun Study. JAMA. 1997;277:813-817.

\begin{tabular}{ll}
\hline KARGER & (c) 2009 S. Karger AG, Basel \\
0251-5350/09/0334-0305\$26.00/0 \\
$\begin{array}{l}\text { Fax +4161306 1234 } \\
\begin{array}{l}\text { E-Mail karger@karger.ch } \\
\text { www.karger.com }\end{array}\end{array}$ & $\begin{array}{l}\text { Accessible online at: } \\
\text { www.karger.com/ned }\end{array}$
\end{tabular}




\section{Stroke: Evidence-Based Management} and Epidemiology Update

\section{Peter Rothwell}

University Department of Clinical Neurology, University of Oxford, Oxford, UK

The session on stroke evidence-based management centered on the evolution of treatment of carotid stenosis, particularly the use of carotid endarterectomy (CEA) and carotid artery stenting (CAS).

CEA has been around since the 1950s. The 2 big trials in endarterectomy for symptomatic carotid stenosis, the NASCET [1] and the ECST [2], showed that CEA reduced the risk of stroke in patients with severe symptomatic carotid stenosis. However, the cut point for benefit differed between the trials, being greater than $50 \%$ stenosis in NASCET and greater than $80 \%$ in ECST. In addition, the degree of stenosis was measured differently in the 2 trials. Furthermore, the outcome events were defined differently. At that stage, there were also no reliable data on clinical, angiographic or pathologic subgroups in which risks and benefits of CEA might differ from the average.

In 2003 and 2004, the Carotid Endarterectomy Trialists' Collaboration worked on combining the NASCET and ECST trials plus a smaller Veteran's Affairs trial. When all individual patient data were reviewed, outcomes redefined and angiograms reassessed, the results of the 2 trials were remarkably similar [3, 4]. Benefit from CEA was substantial for severe stenosis (70$99 \%$ ), significant for moderate stenosis (50-69\%), but absent for lesser degrees of stenosis and for patients with near-occlusion. Subgroup analyses showed the benefits of surgery were greater in men than women, increased with age, decreased with time since last event, were greater for cerebral events versus ocular events and in cases with irregular or ulcerated plaques.

The subgroup analyses are of some help in terms of targeting treatment, developing risk tables and understanding when surgery is clearly indicated. However, the optimal timing of CEA remains uncertain. The analyses of the trial data showed that benefit was much greater if surgery was done within 2 weeks of the presenting transient ischemic attack (TIA) or minor stroke. The risk of stroke or minor TIAs is very high in the first few days, but there are no trial data on the risks and benefits of urgent surgery. However, data on the operative risk are available from surgical case series. An update of a previous systematic review [5] recently showed that in neurologically stable patients with recent TIA or nondisabling stroke, there was little difference in operative risk between early versus late CEA. On balance, operating within a day or two of a TIA or minor stroke is sensible, but more data are required in patients with more substantial cerebral infarction. In reality, however, we aren't meeting a 2-week cutoff for time to CEA. A recent UK National Audit [6] showed that only about $20 \%$ of patients had a CEA within 2 weeks after initial event.

Available since the early 1990s, CAS has really taken off as an alterative to CEA, particularly in the US. In theory, CAS makes very good sense, patients often seem to like it, it is less invasive than open surgery, and stenting works well in peripheral vasculature.

However, data from large trials, such as (SPACE [7] and EVA-3S [8]), suggest that even with protection devices, the procedural risks of CAS are still quite high. Preliminary results from more recent trials confirm this and there is increasing evidence of very high rates of silent brain infarction on post-procedure imaging. Current evidence suggests that CAS can be used in re-stenosis after CEA, post-radiotherapy stenosis, cases of high stenosis/bifurcation, and perhaps if arterial angiography is required anyway. Patient choice and other subgroups may also affect for whom CAS is indicated.

For patients with asymptomatic stenosis, the absolute benefits of CEA are small. CEA appears to be less effective in women than men, as women tend to have more benign fibrous plaques. Recent long-term follow-up data out to 10 years from the Asymptomatic Carotid Surgery Trial (ACST) showed that the absolute benefit from treatment did not increase over time, the absolute risk reduction for disabling or fatal stroke after CEA being very small at 10 years, requiring about 70 patients to have CEA to prevent one stroke.

The major change in the last 15 years has been the improvements in medical treatment, particularly the use of statins. Natural history studies are now showing a much lower risk of ischemic stroke distal to $50-99 \%$ asymptomatic carotid stenosis than was the case at the time that the large trials were done. In the trials in the early 1990s, few of the patients were on statins; a much lower potency and dose of statins was in use in the early 1990s. With an $80 \mathrm{mg}$ simvastatin equivalent, medical treatment might now be better than either CAS or CEA. The results from ongoing interventional trials with medical arms and further work are needed to address many of the issues raised.

\section{References}

1 Barnett HJ, Taylor DW, Eliasziw M, Fox AJ, Ferguson GG, Haynes RB, Rankin RN, Clagett GP, Hachinski VC, Sackett DL, Thorpe KE, Meldrum HE, Spence JD: Benefit of carotid endarterectomy in patients with symptomatic moderate or severe stenosis. North American Symptomatic Carotid Endarterectomy Trial Collaborators. N Engl J Med 1998;339:14151425.

2 Randomised trial of endarterectomy for recently symptomatic carotid stenosis: final results of the MRC European Carotid Surgery Trial (ECST). Lancet 1998;351:1379-1387.

3 Rothwell PM, Eliasziw M, Gutnikov SA, Fox AJ, Taylor DW, Mayberg MR, Warlow CP, Barnett HJ; Carotid Endarterectomy Trialists' Collaboration: Analysis of pooled data from the randomised controlled trials of endarterectomy for symptomatic carotid stenosis. Lancet 2003;361:107-116.

4 Rothwell PM, Eliasziw M, Gutnikov SA, Warlow CP, Barnett HJ; Carotid Endarterectomy Trialists Collaboration: Endarterectomy for symptomatic carotid stenosis in relation to clinical subgroups and timing of surgery. Lancet 2004;363:915-924.

5 Rerkasem K, Rothwell PM: Systematic review of the operative risks of carotid endarterectomy for recently symptomatic stenosis in relation to the timing of surgery. Stroke 2009;40:e564-e 572.

6 UK National CEA Audit Group: Waiting times for carotid endarterectomy in UK: observational study. BMJ 2009;338:b1847.

7 SPACE Collaborative Group, Ringleb PA, Allenberg J, Brückmann H, Eckstein HH, Fraedrich G, Hartmann M, Hennerici M, Jansen O, Klein G, Kunze A, Marx P, Niederkorn K, Schmiedt W, Solymosi L, Stingele R, Zeumer H, Hacke W: 30 day results from the SPACE trial of stent-protected angioplasty versus carotid endarterectomy in symptomatic patients: a randomised non-inferiority trial. Lancet 2006;368:1239-1247.

8 Mas JL, Chatellier G, Beyssen B, Branchereau A, Moulin T, Becquemin JP, Larrue V, Lièvre M, Leys D, Bonneville JF, Watelet J, Pruvo JP, Albucher JF, Viguier A, Piquet P, Garnier P, Viader F, Touzé E, Giroud M, Hosseini H, Pillet JC, Favrole P, Neau JP, Ducrocq X; EVA-3S Investigators: Endarterectomy versus stenting in patients with symptomatic severe carotid stenosis. NEJM 2006;355:1660-1671. 
Parkinson's Disease: Evidence-Based Medicine and Epidemiological Update

\section{Amos D. Korczyn \\ Sieratzki Chair of Neurology, Tel-Aviv University Medical School, Ramat Aviv, Israel}

Parkinson's disease (PD) is the second commonest disease in adults $>65$ years after Alzheimer's disease (AD) and is clinically seen as tremor, rigidity and/or bradykinesia; however, little consistent data exists.

Two hundred years ago, the London physician James Parkinson described the 'shaking palsy'. In the 1860s, Jean-Martin Charcot widened the scope and stressed that not all Parkinson's patients shake. Current data on the prevalence and incidence of PD remains unreliable, partly because $\mathrm{PD}$ is poorly recognized compared to AD. Roughly, the prevalence in $>65$ years is $1.5 \%$ with worldwide approximately 7 million people with $\mathrm{PD}$; the overall incidence is about 2.5 to 4 cases per 100,000 (unadjusted).

The ratio of incidence to prevalence is indicative of the survival or duration of disease with respect to its mortality. As these measures are defined as events in 1 year (365 days), if the prevalence is near or equal to incidence, the duration of the disease would be 1 year. The prevalence of PD is about 10 times greater than the incidence, meaning survival is about 10 years. This has implications to the definitions and epidemiological questions regarding $\mathrm{PD}$ etiology.

Consider for the moment that PD can be characterized as a chronic, progressive neurodegenerative disease that has a slow, relatively selective loss of tissue from the substantia nigra, which gradually loses its function to release essential neurotransmitters that control movement and coordination. The accepted definition is that 2 or more major manifestations must be present (tremor at rest, rigidity or bradykinesia, in the absence of a secondary cause). However, the extended duration and likely temporal difference in observing a probable manifestation of PD implies the actual 'onset' is not known or should be dated backwards.

Another challenge for epidemiologists and clinicians is whether similar pathologies should be split or lumped together with PD. These syndromes or 'parkinsonisms' include: progressive supranuclear palsy, multiple system atrophy, corticobasal ganglionic degeneration and essential tremor, among others. There are indicators that these syndromes could be split based on etiologic, pathological, clinical, therapeutic, or even genetic lines.

The following etiologies have been proposed: infective (e.g. encephalitis lethargia), toxic (MPTP, $\mathrm{Mn}^{++}$; neuroleptics, i.e. treatment-induced parkinsonism), traumatic (Boxer's parkinsonism; traumatic brain injuries in younger persons), as well as vascular, genetic and other degenerative disorder causes.

According to the pathology, Lewy bodies (LB) are the hallmark of PD. The presence of LB is seen in sporadic PD, some but not all genetic PD (Parkin, LRRK2 genes) and is not present in toxic or post-encephalitic parkinsonism. This heterogeneity suggests that LB can not be truly considered a marker of PD pathogenesis. Furthermore, PD does not mean the presence of LB exclusively and can involve other brain pathology (e.g. intraneuronal lesions, axons and dendrites). Additional stains identify $\alpha$-synuclein and ubiquitin inclusions. Plausibly, any abnormal protein deposition may lead to neuronal insufficiency. Synucleinopathies, tauopathies, amyloidopathies, and other pathological proteins could provide additional classifications, the clinical utility of which need to be better understood.

Discrepancies between clinical and pathological findings have been illustrated. Furthermore, 'pre-motor' or non-motor clinical symptoms may also be brain-related such as pain, depression (before nigra involvement), constipation, anosmia, REM sleep behavior disorders, periodic limbic movements of sleep, and perhaps even weight loss. When Parkinson described the 'senses and intellect were unaffected', we know now this is not true. All 'shaking palsy' patients will develop dementia eventually and cases of dementia with PD before motor involvement exist.

A brief review of the types of dementia provides more information but not necessarily more evidence. For instance, is dementia with LB (DLB) a real disease - different from dementia which occurs in advanced PD - or are these all possibilities on a spectrum? PD is clearly heterogeneous and the continuum concept of disease is under big debate.

The epidemiological data suggests that the pre-clinical stage of PD can be up to 20 years. How is this dealt with in epidemiological study designs when the 'disease-free' controls may not be so clear cut? The crux of how disease is defined crops up again and again. Measurements are what they are and depend on the definition, which is imprecise and is not the whole truth.

Classical cases consider tremor and DLB to be dominant. Some other non-classical PD cases useful to keep in mind are the akinetic-rigid forms, pure autonomic failure (although parasympathetic involvement possible) and the clinical aspects (e.g. constipation). To date, there are no biological markers of vulnerability, disease progression, or therapy response in $\mathrm{PD}$, due possibly to the heterogeneity of the disease. For all these in between cases, there is no good evidence for management.

In conclusion, PD and its related syndromes are complex, heterogeneous neurodegenerative diseases with multifactorial etiology. Although controversial, the concept of PD as a spectrum is gaining strength and needs to be further investigated with regards to its clinical utility.

\section{Epilepsy: Evidence-Based Medicine and Epidemiological Update}

\section{Ettore Beghi}

Capo Laboratorio Malattie Neurologiche, Istituto "Mario Negri", Milano, Italy

In this session, the available meta-analyses on the prevention of seizures and epilepsy, relapse and treatment of the first seizure, efficacy and safety of first and second generation antiepileptic drugs (AEDs), as well as treatment discontinuation are reviewed. Although meta-analyses synthesize studies and information together in a sufficiently clear and often understandable format, a meta-analysis of small trials can never substitute for a large randomized controlled trial (RCT). Meta-analysis decreases alpha error (difference interpreted as a chance finding) and beta error (no difference interpreted as equivalence). In this way, meta- 
analysis offers reliable evidence in the absence of definitive clinical trials.

Biases and the individual deficiencies of studies are often incorporated in meta-analyses, a major drawback. In addition, new sources of bias can be introduced (e.g. selection of published studies) and the heterogeneity of pooled studies can be overlooked to the detriment of the meta-analysis ('mixing apples and oranges'). Moreover, meta-analyses do not have the statistical power that replaces the randomization and power of a large sample population. Clinical studies on the prevention of epileptogenesis show conflicting results, thus, no strong conclusions can be made. In patients with moderate-to-severe head trauma, the prophylactic use of AEDs is successful for the prevention of provoked (acute symptomatic) seizures, but not for the prevention of epilepsy (i.e. repeated unprovoked seizures). Complications of treatment include skin rash (severe enough to stop treatment). Results are similar in prevention trials for other clinical conditions characterized by provoked and unprovoked seizures.

This apparent lack of efficacy of AEDs for the prevention of unprovoked seizures may be explained by the inappropriate study sample, the use of an inactive drug, the failure to adjust for confounders, or the insufficient power of the studies. Further research is needed with larger and/or selected sample populations. There is not sufficient evidence to support AED prophylaxis in unprovoked seizures at this time.

In observational studies, the risk of recurrence of a first unprovoked seizure is $27-71 \%$. Several prognostic predictors are implicated and treatment of the first seizure may affect the risk of recurrence (up to $42 \%$ relapse within 2 years in a meta-analysis). A documented etiology, an abnormal electroencephalogram (EEG) [1], sleep and partial seizures and family history of seizures can predict recurrence risk. There is weak or no evidence to show that prior provoked seizures, age, sex, status epilepticus, post-ictal paresis, or abnormal neuro- or psychological findings are predictors. In addition, the treatment of a first unprovoked seizure is associated with a reduced risk of relapse only during the first years of follow-up [2]. In clinical practice, patients may be less compliant to take treatment due to known side effects. Currently, no meta-analysis is available on the effects of treatment on the long-term prognosis of epilepsy.

A number of second generation AEDs has been marketed in the last 20 years. Yet most of these RCTs do not provide any insight how new AEDs compare to first generation treatments. As most of the drugs show efficacy versus placebo in terms of probability of reducing seizure frequency by $50 \%$, it is interesting to look at the retention time. This is how much time the patient stays on the study drug and it is an indirect measure of drug tolerability, as most withdrawals are due to adverse effects. With the exception of gabapentin (GBP), levetiracetam (LEV) and lamotrigine (LTG), the new AEDs - oxcarbazepine (OXC), pregabalin (PGB), tiagabine (TGB), topiramate (TPM) and zonisamide (ZNS) have a greater tendency for more withdrawals due to study drug. Common adverse events due to study drug include ataxia, dizziness, fatigue, nausea / vomiting, somnolence.

In a meta-analysis, carbamazepine (CBZ) and valproic acid (VPA) were comparable both in the control of partial seizures and control of generalized seizures which is in conflict to clinical practice. This is a problem of the meta-analysis whose results may be in part explained by a misdiagnosis of newly diagnosed patients at inclusion. In addition, barely significant and opposing results reflect the heterogeneity of the studies included in the review and consequently a poor analysis, giving a false interpretation.

Second generation AEDs (i.e. LTG, TPM, OXC) have been consistently shown to be effective in partial epilepsies (studied more systematically and accurately) as an add-on treatment versus placebo. However, there is no clear evidence of differences between the first generation AEDs (i.e. phenytoin, phenobarbital, CBZ, VPA) for the treatment of partial and generalized epilepsies. Different designs, sample sizes, the heterogeneity of study populations and misclassification of seizures and epilepsies may all hamper the results of meta-analyses.

After treatment discontinuation, there is a large range of relapse rates. Older age, abnormal EEG and documented etiology predict a higher risk of relapse; timing of withdrawal and tapering rate are also important. The meta-analysis of the probability of remaining seizure free after withdrawal gave a $71 \%$ rate at 2 years, from studies in children and adults [3]. The risk factors for relapse were statistically significant but not clinically relevant. Based on these data, one could still consider withdrawing a drug based on EEG abnormalities and documented etiology.

There is a higher probability to remain seizure-free after withdrawal if the interval since the last seizure is longer than 2 years. In children, treatment discontinuation after $<2$ years of seizure freedom is associated with a higher recurrence risk than discontinuing after $>2$ seizure-free years. A solitary study was available on AEDs tapering; however, due to the methodological deficiencies and small sample size, no reliable conclusions can be identified regarding the optimal rate.

It is important to note that a meta-analysis can not provide good synthesis and information on incidence, risk factors, or prognosis and, in this regard, it should not be the basis for a clinical guideline. The heterogeneity of design and results of these kinds of studies is very wide. It is hoped the Cochrane Collaboration can provide systematic analyses for diagnostic studies (sensitivity, specificity, etc.) and for the study of risk factors in the future.

\section{References}

1 Berg AT, Shinnar S: The risk of seizure recurrence following a first unprovoked seizure: a quantitative review. Neurology 1991;41:965-972.

2 Wiebe S, Téllez-Zenteno JF, Shapiro M: An evidence-based approach to the first seizure. Epilepsia 2008;49(Suppl 1):50-57.

3 Berg AT, Shinnar S: Relapse following discontinuation of antiepileptic drugs: a meta-analysis. Neurology 1994;44:601-608.

\section{Multiple Sclerosis: Evidence-Based Medicine and Epidemiological Update}

\section{Jürg Kesselring \\ Department of Neurology and Neurorehabilitation, Rehabilitation Center, Valens, Switzerland}

Clinical manifestations in multiple sclerosis are well described; however, guidelines on management are only starting to be developed [1]. Most rehabilitation programs focus on improving the patient's quality of life and overall function as well as providing data towards public health policies, e.g. towards improving the 
infrastructure to accommodate affected individuals. In particular with multiple sclerosis, optimal therapy and management should include the patient's active participation.

The diary of Augustus Frederick d'Este (1794-1848) provided one of the earliest documented example of multiple sclerosis. It is now known that multiple sclerosis is not psychosomatic in origin, although the myth exists and some patients are convinced it is 'just stress'. The clinical manifestations of the disease - fatigue and temperature lability; bladder, bowel and sexual dysfunction (so-called pelvic multiple sclerosis); poor dexterity and motor function; dizziness, visual disturbances, difficulties in speech and swallowing; and associated pain - need to be carefully recognized through appropriate questions at examination. Newly diagnosed patients especially need good and reliable information about this long-lasting chronic disease with a waxing and waning disease course.

Fatigue, differentiated from depression, occurs in more than half of patients. For many, fatigue is the most disabling symptom and can occur at any stage. It is described as a 'central' fatigue that has no correlation to sleep deprivation or known causes of fatigue (medications, feeling tired, depression, etc.) This central fatigue can be from nocturia, pain, or spasticity. There is some evidence to suggest that there is an immunological response (e.g., TNF- $\alpha$, IL-1, leptin); other evidence suggests alterations in the hypothalamus-pituitary axis in the pathogenesis.

Motor problems over time range from disuse and deconditioning. Strength, force, endurance and range of motion should be evaluated and improved. Patients with inadequate motor recruitment of spinal motor units have an inability to drive motor neurons. This can be seen with functional MRI - more brain is used for the same effort, leading to more energy spent and more fatigue in a vicious circle [2]. Movement is akin to 'running twice the distance' due to spastic and antagonistic muscle contractions.

General measures to manage motor problems include teaching patients to economize forces, treating bladder disturbances, spasms, periodic limb movements of sleep or pain such that patients can get undisturbed regular sleep, avoiding or discontinuing fatigue-inducing medicines or substances, and lastly, optimizing performance capacity by getting regular exercise.

Because of the myelin loss, there is a blocking temperature that is reached earlier in patients with multiple sclerosis. Studies of conduction velocity versus temperature have demonstrated this temperature dependency, known as the Uthoff-phenomenon. The experience in Switzerland is to use full-body ice baths or cooling garments to help patients extend performance capacity.

Medications available for motor and circulation dysfunction are not as useful as active participation and can have untoward effects. Other less effective treatments include pulsed magnetic resonance therapy; a blinded randomized controlled trial using this technique showed that there was no difference between having an active versus non-active mattress [3]. Clearly, the MS patient must remain active as passive or pharmacological treatments are not as effective.

Frequent micturition (urgency, frequency and hesitancy) is an issue for nearly every patient with multiple sclerosis and thus, he or she needs a socially acceptable way to void. Studies indicate that in 1 hour, at least 15 minutes is spent by the patient towards micturition. Evidence regarding urge incontinence implicates the pontine micturition center [4], thus, physical and mental origin need to be differentiated. Bladder training may be possible; how- ever, for most patients, medications, self-catheterization, or permanent in-dwelling catheters can work. The aim for the patient is to have a life not centered on micturition.

Sexual dysfunction is common, although it may not be easy for the patient to communicate this. Counseling, oral medication, intraurethecal pharmaco-therapy and other physical intrusive methods should be discussed with male patients. Another silent symptom is chronic constipation. It can be a result of immobility, nutrition or medications and needs to be uncovered and addressed promptly.

We know now that the concept of the 'premorbid personality' from earlier time is nonsense. Although there may be clinical neuropsychological issues, they first should be considered as a natural reaction to the frustrations experienced. Crying, anger, fear, hopelessness are all valid feelings and emotions; recognizing and helping the patient develop coping skills is vital to prevent these feelings from becoming pathologic. Psychological reactions, cognitive dysfunction, affective and emotional disturbances need to be recognized and treated; selective serotonin reuptake inhibitors are usually helpful.

Rehabilitation includes an adjustment process to the unpredictable and uncertain nature of multiple sclerosis, which clinicians need to appreciate and try to see from the patient's shoes. This goes well beyond evidence-based medicine and it should be the perspective of any clinician working with patients with multiple sclerosis.

\section{References}

1 Kesselring J, Beer S: Symptomatic therapy and neurorehabilitation in multiple sclerosis. Lancet Neurology 2005;4:643-652.

2 Pantano P, Mainero C, Caramia F: Functional brain reorganization in multiple sclerosis: evidence from fMRI studies. J Neuroimaging 2006;16:104-114.

3 Mostert S, Kesselring J: Effect of pulsed magnetic field therapy on the level of fatigue in patients with multiple sclerosis-a randomized controlled trial. Mult Scler 2005;11:302-305.

4 Blok BF, Sturms LM, Holstege G: Brain activation during micturition in women. Brain 1998;121(Pt 11):2033-2042.

\section{Migraine: Epidemiology and Evidence-Based Management Update}

\section{Hans-Christoph Diener \\ Department of Neurology and Headache Center, University Duisburg-Essen, Essen, Germany}

Headaches are an important challenge in neurology as $20 \%$ of outpatient visits to neurologists in private practice cover migraine and other headaches. The 1-year prevalence is highest for episodic tension-type headaches ( $40 \%$ overall, equal among males and females), followed by migraines (18\% in females, $6 \%$ in males).

Globally, with the exception of Asian countries, most epidemiological studies show similar incidence and prevalence. Migraine has an age- and gender-specific incidence, prevalence and age of onset (incidence 2:1 female:male ratio). The higher incidence, severity and frequency in women may be explained by genetic factors and fluctuations in hormones. Interestingly, prevalence and conditions peak then improve after age 40 . 
Established significant co-morbidities of migraine include depression, anxiety disorder, hypertension, epilepsy, ischemic stroke, Raynaud's syndrome, allergies and chronic lower back pain. In the last 5 years, additional co-morbidities have been recognized: obesity, fibromyalgia (mainly in women), irritable bowel syndrome, syncope, myocardial infarction and endometriosis. Headache and gastrointestinal complaints are common. Several large population-based studies also showed that headaches are associated with reflux, diarrhea, constipation and nausea.

Strong evidence shows migraine without aura is not associated with increased risk of ischemic stroke. Migraine with aura is a risk factor for ischemic stroke. The overall relative risk of stroke is 2.16 in all patients experiencing migraine (meta-analysis of 3 cohort and 11 case-control studies) [1]. Furthermore, migraine with aura may also be a marker for increased risk of ischemic coronary events. However, the mechanisms by which migraine with aura may lead to ischemic vascular events are likely complex and may differ for ischemic stroke and myocardial infarction [2, 3]. Further research is needed to identify individuals with migraine with aura who are at particular increased risk of cardiovascular disease.

In prospective population-based studies, chronic tension-type headache in Germany has a 1-year incidence at 3\% (defined as $>15$ days per month). The major risk factors for the transition from episodic to chronic headache ('chronification') are female sex, single, snoring, head or neck trauma, negative life events, major depression, social class, obesity, other chronic pain, medication overuse and anxiety disorders. Unfortunately, chronic headache patients are referred to the specialist when it is already too late.

Chronic tension-type headache pathophysiology appears to be different from medication overuse, migraine patients and individuals with no headache history, based on an imaging study [4]. In the chronic headache patients, gray matter was significantly decreased in regions known to be involved in pain processing and can be considered irreversible. Migraine is a different disease than chronic tension-type headache and it is much more important to identify these patients before the headache becomes chronic. One way to find these individuals is to use these risk factors in an algorithm and together with headache codes find them in patient databases from insurance companies. In Germany, the Headache Center collaborates with insurance companies who have this data, and a large proportion of our patients are recruited this way.

For the acute treatment of migraine, the most effective drugs are clearly the triptans; however, there is no real difference among the many triptans available regarding which is the most effective [5]. It is trial and error to find out which triptan works best for which patient. Simple and combination analgesics are established therapies often taken together with antiemetics [6]. Studies also showed that cutaneous allodynia is not a predictor of non-response to acute treatment with triptans.

Acute treatment with a triptan should occur as early as possible (2-hour pain-free success rate: in mild attacks, 53\% but in moderate-severe, $37 \%$ ) and only in patients who can tell the difference between migraine and tension headache. The acute treatment works best in infrequent migraine patients; otherwise there is a chance of medication overuse. The combination of sumatriptan plus naproxen showed 2-hour responses and 24-hour pain relief to be significantly higher with the combination than either agent alone or placebo [7]. Combinations are quite common in practice and can be used to save over-medication with triptans.
Calcitonin gene-related peptide antagonists (e.g. telcagepant) in the treatment of acute migraine attacks have shown to be as effective as triptans [8]. The advantages of this new class in acute migraine treatment are it has no vasoconstrictive properties, can be used by patients for whom triptans are contraindicated and has a safety profile similar to placebo [9]. It would be interesting to know how telecagepant would work in combinations with triptans or NSAIDs.

For patients with frequent migraine attacks, prevention would be ideal. However, there is no clear scientific background to use common prophylaxis treatments or why they work in some patients (e.g. good evidence: topiramate, valproic acid, beta blockers, flunarizine; weak evidence: magnesium, NSAIDs, vitamin $\mathrm{B}_{2}$, etc.). The list is not comprehensive and non-medical management has also been reported (acupuncture, exercise). Epidemiological evidence shows that regular exercise is correlated with fewer headache days [10].

However, what happens after preventative therapy is stopped? The next study looked at people titrated with topiramate openlabel after 6 months. In some patients, migraine does get worse (rebound), but $80 \%$ were still better off with initial prophylaxis after termination [11]. This study shows that the reduction of migraine days achieved by effective migraine prophylaxis is a least partly maintained after termination of prophylaxis at 6 months. Prophylactic treatment with oxcarbazepine [12], divalproex sodium in adolescents [13], and estrogen in menstrual migraine [14] were not effective in migraine prophylaxis.

The third most common type of headache is from medication overuse and is mostly seen in Headache Centers. Epidemiological studies worldwide indicate that the prevalence ranges from $1-4 \%$ [15]. This may be in part due to how medication is delivered. For example, in Norway patients must pay out of pocket, thus medication overuse is much more difficult. Another study suggests that migraine preventive therapy with topiramate should be offered to patients with chronic migraine with and without medication overuse headache $[16,17]$.

Successful treatment of difficult headache patients can be achieved by integrated care from neurologists / pain specialists, behavioral psychologists, physical therapists and sports therapists, with each contributing to the diagnosis and care, including connected systems and collaboration with insurance companies. It is important to understand that migraine is a reversible condition whereas chronic tension-type headaches are not. The information gained from epidemiological studies enables one to identify those people who can be helped by a particular approach.

\section{References}

1 Etminan M, Takkouche B, Isorna FC, Samii A: Risk of ischaemic stroke in people with migraine: systematic review and meta-analysis of observational studies. BMJ 2005;330:63-65.

2 Bigal ME, Kurth T, Hu H, Santanello N, Lipton RB: Migraine and cardiovascular disease: possible mechanisms of interaction. Neurology 2009;72: 1864-1871.

3 Kurth T, Schurks M, Logroscino G, Gaziano JM, Buring JE: Migraine, vascular risk, and cardiovascular events in women: prospective cohort study. BMJ 2008;337:a636.

4 Schmidt-Wilcke T, Leinisch E, Straube A, Kampfe N, Draganski B, Diener $\mathrm{HC}$, et al: Gray matter decrease in patients with chronic tension type headache. Neurology 2005;65:1483-1486.

5 Ferrari MD, Roon KI, Lipton RB, Goadsby PJ: Oral triptans (serotonin 5-HT1B/1D agonists) in acute migraine treatment: a meta-analysis of 53 trials. Lancet 2001;358:1668-1675. 
6 Evers S, Afra J, Frese A, Goadsby PJ, Linde M, May A, et al: EFNS guideline on the drug treatment of migraine-revised report of an EFNS task force. Eur J Neurol 2009;16:968-981.

7 Brandes JL, Kudrow D, Stark SR, O'Carroll CP, Adelman JU, O’Donnell FJ, et al: Sumatriptan-naproxen for acute treatment of migraine: a randomized trial. JAMA 2007;297:1443-1454.

8 Connor KM, Shapiro RE, Diener HC, Lucas S, Kost J, Fan X, et al: Randomized, controlled trial of telcagepant for the acute treatment of migraine. Neurology 2009;73:970-977.

9 Olesen J, Diener H, Husstedt IW, Goadsby PB, Hall D, Meier U, et al: Calcitonin gene-related peptide (CGRP) receptor antagonist BIBN4096BS is effective in the treatment of migraine attacks. N Engl J Med 2004; 350:1104-1110.

10 Varkey E, Hagen K, Zwart JA, Linde M: Physical activity and headache: results from the Nord-Trondelag Health Study (HUNT). Cephalalgia 2008;28:1292-1297.

11 Diener HC, Agosti R, Allais G, Bergmans P, Bussone G, Davies B, et al: Cessation versus continuation of 6-month migraine preventive therapy with topiramate (PROMPT): a randomised, double-blind, placebo-controlled trial. Lancet Neurol 2007;6:1054-1062.

12 Silberstein S, Saper J, Berenson F, Somogyi M, McCague K, D’Souza J: Oxcarbazepine in migraine headache: a double-blind, randomized, placebo-controlled study. Neurology 2008;70:548-555.

13 Apostol G, Cady RK, Laforet GA, Robieson WZ, Olson E, Abi-Saab WM, et al: Divalproex extended-release in adolescent migraine prophylaxis: results of a randomized, double-blind, placebo-controlled study. Headache 2008;48:1012-1025.

14 MacGregor EA, Frith A, Ellis J, Aspinall L, Hackshaw A: Prevention of menstrual attacks of migraine: a double-blind placebo-controlled crossover study. Neurology 2006;67:2159-2163.

15 Diener HC, Limmroth V: Medication-overuse headache: a worldwide problem. Lancet Neurology 2004;3:475-483.

16 Silberstein SD, Lipton RB, Dodick DW, Freitag FG, Ramadan N, Mathew $\mathrm{N}$, et al: Efficacy and safety of topiramate for the treatment of chronic migraine: a randomized, double-blind, placebo-controlled trial. Headache 2007;47:170-180.

17 Diener HC, Bussone G, Van Oene J, Lahaye M, Schwalen S, Goadsby P: Topiramate reduces headache days in chronic migraine: a randomized, double-blind, placebo-controlled study. Cephalalgia 2007;27:814-823.

\section{Amyotrophic Lateral Sclerosis: Epidemiology and Evidence-Based Management Update}

\section{Giancarlo Logroscino}

Department of Neurology and Psychiatry School of Medicine, University of Bari, Bari, Italy

Many challenges exist in characterizing amyotrophic lateral sclerosis (ALS) due to the low incidence, phenotypic variability, lack of a biological marker and uncertainty in the diagnostic criteria. Before the early 1990s, only observational studies, mainly based in clinical centers, were available. In the last 20 years several population-based registries were developed in Europe with an approach similar to cancer registries. Registries are useful for gaining knowledge on the frequency and natural history of the disease, can provide clinical insight on specific phenotypes and data-driven improvements towards the care of ALS patients.

Registries are characterized by having a geographically defined population, defined multiple sources of case ascertainment, and a standardized, prospective way of collecting data based on uniform diagnostic criteria (i.e. El Escorial Criteria, EEC). The disadvantage of using registry data, particularly in ALS, lies in the low power of the analysis as a result of the low incidence. The average output for most of registries based in Europe, is only 60 to 80 ALS cases per year.

The objective of complete case ascertainment is achievable with a thorough definition of the geographical coverage. The ongoing Puglia registry (Italy) is a longitudinal study on the incidence, natural history and risk factors in ALS. This registry includes 23 neurological facilities, with different characteristics (neurological departments, neurophysiological units with electromyography) that include all the possible gates through which a subject with a diagnostic suspicion of ALS should go. This approach is the most likely way to have a complete geographical coverage of the area of interest (Puglia with a population of about 4 million). This approach is simpler in countries with a national health system, and thus, has a single provider of care as in all western European countries. This kind of registry would be nearly impossible to set up in the US, where the presence of multiple health providers even in small areas makes impossible or extremely difficult to collect data in a homogenous way.

It is important to understand the effect of referral and be able to recognize selection bias in the data. One study [1] showed outpatients were different from inpatients - inpatients were more likely older, female, and had a shorter duration of disease at diagnosis, which carries a poor prognosis. This selection bias was confirmed in EU studies [2].

The ongoing prospective EURALS project registry also has produced useful information. EURALS has a 24 million reference population from Lombardia, Puglia, Piedmonte (Italy), Scotland, Ireland, and Lancashire (UK); the registry has identified that incident ALS cases are at about 1,000. The mean age of onset in this population was 65 years, and the male:female ratio is almost 1 (at the moment $>1$ ). Patients identified in this registry are older than in most clinical trials of ALS (56 or 57 years) [3].

As the period between the onset of ALS and diagnosis is typically closer to 15 months, this might mean that some people are being counted incorrectly; this does not, however, affect the denominator. In the EURALS registry, the incidence per 100,000 people is 2.16 (males 2.4, females 1.95). Interestingly, the incidence peaks at 70-74 years, which is different from earlier epidemiological studies, where the peak ALS incidence was at around 50-54 years.

Part of this shift may be due to the understanding of the disease. The classical way of looking at ALS as solely a disease of the brain stem - with specific anatomical and pathological characteristics - is slowly changing, based on complex evidence. From the data on the anatomical localization of affected nerves (spinal versus bulbar/generalized ALS), both gender- and age-related differences can be observed. The spinal ALS cases influence the relationship to age, which cause the overall incidence to shift to the right (increasing with age).

EURALS data show spinal onset is more prominent in Italy, whereas bulbar is more prominent in Ireland. Is there a difference in the clinical definition of the phenotype or are these differences due to a real al difference in the clinical phenotype at onset in the 2 areas? This 'hidden' evidence of ethnic variation was found when the analysis was restricted, i.e. to the age group where the ALS diagnosis is most accurate (in 45-75 year-old patients). 
Registry data are however imperfect and up today, did not provide data on risk factors of the disease. Large cohort studies are better suited to collect data to explore the role of possible risk factors. These are observational data and differences in delivery of care, or in the answers to self-reported questionnaires and other uncontrolled factors, may bias the data. This is particularly important when trying to assess risk factors. However, strong evidence does not exist for any (non-geographical) exposure. Excessive or inappropriate strenuous physical activity, combined with a stressimpacting profession, body habitus, and trauma have all been implicated as possible exposures that may increase the risk of developing ALS.

The challenge is the time factor; with ALS, the induction period can be very long. Gulf War data showed that young US military veterans had an excess incidence of ALS after observing the veterans over 7 years after exposure [4]. The results of this study, confirmed in other studies on the same veteran cohort, were instrumental to identify these veterans as a probable cluster and helped recognize war as a possible environmental exposure.

Symptomatic management is the current best practice, with most drugs being ineffective. Riluzole has modestly slowed down the progression of disease in randomized controlled trials. In recent years, percutaneous gastrostomy (PEG) and non-invasive ventilation (NIV) have been shown to improve survivorship and quality of life in several studies. However, in a population-based setting, there is no improvement in survival when ALS mortality is compared in the period pre- and post- introduction of riluzole, PEG and NIV [5]. Clearly, prospective studies and further work is needed on the clinical management and therapy side, aimed at improving social function and general health in patients with ALS.

\section{References}

1 Lee JR, Annegers JF, Appel SH: Prognosis of amyotrophic lateral sclerosis and the effect of referral selection. J Neurol Sci 1995;132:207-215.

2 Logroscino G, Traynor BJ, Hardiman O, Chiò A, Couratier P, Mitchell JD, Swingler RJ, Beghi E; EURALS: Descriptive epidemiology of amyotrophic lateral sclerosis: new evidence and unsolved issues. J Neurol Neurosurg Psychiatry 2008;79:6-11.

3 Logroscino G, Traynor BJ, Hardiman O, Chiò A, Mitchell D, Swingler RJ, Millul A, Benn E, Beghi E: Incidence of amyotrophic lateral sclerosis in Europe. J Neurol Neurosurg Psychiatry 2009 Aug 25. [Epub ahead of print]

4 Horner RD, Kamins KG, Feussner JR, Grambow SC, Hoff-Lindquist J, Harati Y, Mitsumoto H, Pascuzzi R, Spencer PS, Tim R, Howard D, Smith TC, Ryan MA, Coffman CJ, Kasarskis EJ: Occurrence of amyotrophic lateral sclerosis among Gulf War veterans. Neurology 2003;61:742-749.

5 Forbes RB, Colville S, Cran GW, Swingler RJ; Scottish Motor Neurone Disease Register: Unexpected decline in survival from amyotrophic lateral sclerosis/motor neurone disease. J Neurol Neurosurg Psychiatry 2004; $75: 1753-1755$.

\section{Editor's Session: How to Write a Good Article}

V.L. Feigin (Editor-in-Chief, Neuroepidemiology)a

M.G. Hennerici (Editor-in-Chief, Cerebrovascular

Diseases) ${ }^{\mathrm{b}}$

${ }^{a}$ Faculty of Health and Environmental Sciences, Auckland University of Technology, AUT North Shore Campus, Auckland, New Zealand, ${ }^{\mathrm{b}}$ Department of Neurology, University of Heidelberg, Universitätsmedizin Mannheim UMM, Mannheim, Germany

An editor's perspective is useful when learning how to write a good scientific article and getting it published. The first question to ask is: why bother? Good scientific communication is the basis on which researchers and clinicians contribute to the knowledge. Great ideas are worthless if you keep it to yourself.

A well-written, publishable manuscript should have:

- an excellent abstract

- a simple, central 'take home' message

- demonstrated originality

- a flawless study design (well-planned investigation)

- concise and clear language

- well presented and appropriately presented results (balanced if there are negative results, e.g. unexpected toxicities, intervention not advisable in context, etc.)

- been targeted to the journal that the paper should be submitted

At some stage after the completion of an analysis or study, consider whether it is time to publish. Keep in mind any conflicts of interest, the scientific point(s) the work addresses, and how it adds to the body of current knowledge. Particularly in clinical research when the outcome is negative, it may be unethical not to publish. Focusing on a single message at this stage also facilitates journal consideration.

Once a target journal is chosen (or a first choice, second choice, etc.), the drafting should be driven by the journal's 'Author's Instructions' or guidelines; authors should read printed articles and references from this journal should be included in the manuscript if appropriate. Specific requirements of the journal may include: CONSORT reporting checklists or similar guidelines to make sure the publication 1) is complete and transparent in reporting, which aids in its critical appraisal and interpretation, and/or 2) meets standards that improve the quality of research used in healthcare decision-making. Furthermore, following journal guidelines are to the author's advantage and assist in producing concise and clear writing.

At this point, a literature search is helpful to refresh knowledge and give details to what is different about the work. As the manuscript is developed, expect several versions until the key point(s) from your data are crystal clear. It is valuable to ask colleagues outside of your expertise area to look at the manuscript; often medical journals are targeted for a general scientific audience.

It's also time to decide on co-authors. There are guidelines here too: the International Committee of Medical Journal Editors (ICMJE) guidelines are followed by nearly every international medical journal. Familiarize yourself with the current guide- 
lines and the target journal(s) - know if they limit the number of authors or whether there is a contributorship/collaboratorship model for persons that do not fall under the authorship criteria (e.g. on behalf of a Study Group). Authorship may also be key for further funding opportunities. Consider the possibility to share first-authorship with your closest co-worker if both contributed similarly to the work and manuscript.

Next, the title of the manuscript should be eye-catching, informative and conclusive, such that the message is brought up front. Questions in the title should be avoided, unless it is an editorial or the answer is definitive from the publication of your work.

The abstract is absolutely vital to getting the paper noticed and published. Therefore, it should be worked on until it reflects clearly and concisely why the study was done, how it was done (study design), the main results, analyses and main conclusions. Abstract results should contain numbers or figures; if the abstract doesn't inform, the paper won't get read. Further background and aims belong in the Introduction.

The reviewers critically assess the Methods section. If the methodology is considered seriously flawed or there is simply a lack of detail, reviewers might reject the paper. The methods must be clear and transparent, sufficient for another to reproduce the methodology. By following the guidelines and not leaving any important questions unanswered, this section should run 2-3 pages.

In the Results section, use tables and figures to illustrate the data. The text should reflect the interpretation of the data; do not repeat what is already presented in the tables. This saves space and it allows the reader to review the data quickly on its own merit.

One common mistake in the Discussion section is the tense the discussion should always be written in the present tense. It is annoying for the editor and reviewers if this rule is not followed, leading to rejection in a high-standard journal. Have a proactive approach and write about the limitations of the study, with care to compare similar studies ('apples to apples'). It is not necessary to discuss insignificant findings, unless they were unexpected or counterintuitive and are clinically significant. Furthermore, a good familiarity with the literature should be demonstrated here.

Finally, remember most medical journals have a high rejection rate, thus, realistic expectations should be set. An accompanying cover letter should explain in few sentences the major results (originality, validity, relevance) and why this particular journal should publish the data. You should enjoy reviewing/reading it yourself; if necessary, ask a native-speaking person for support. Follow the instructions from the reviewer feedback even if you submit to another journal. Consider that the same reviewer might see your manuscript again (numbers of experts are small in some areas) and be puzzled by an ignorance to reasonable comments.

\section{Alzheimer's Disease}

\section{Carol Brayne}

Department of Public Health and Primary Care, Institute of Public Health, University of Cambridge, Cambridge, UK

Industry-sponsored randomized clinical trials come under an immense amount of scrutiny and pressure has mounted for trans- parency in trial reporting recently. Misrepresentation of the results of trials hurts the pharmaceutical industry when it comes to light, as well as the scientists and doctors who believe that such clinical trials do offer incremental improvement and hope.

In public health, an ethical paradigm exists that checks for poorly designed and reported studies and encourages clear conduct and reporting of research such that the objectives and outcomes are clear, with the healthcare context stated. For example, the STROBE initiative [1] should become instrumental in improving the reporting requirement of observational studies. This requirement is key to being able to synthesise research. This has largely been accepted in the epidemiological and public health research domains but has not had that much impact on the reporting of clinical studies.

In the community, there are, simply stated, 3 types of people with dementia: those coping individuals with 'silent' disease who do not seek attention and whose social circumstances support them; those who may have 'silent' disease but also have comorbidities and seek medical attention - a dementia diagnosis in this setting is often a chance finding since we know dementia and depression are poorly recognized in acute clinical settings (as they are not the most important factor in the acute state); and finally, those in whom the disorder is not 'silent' but causes a perturbation in themselves or their environment, not necessarily due to cognitive effects and who tend to, at least in the UK, be referred to the old age psychiatric services. In addition, there are major national differences in community awareness, health and social care delivery to older people with suspected depression or dementia.

These reveal some of the real difficulties in collecting and interpreting data relevant to the whole population. For the purposes of meaningful synthesis of existing research there needs to be a consensus - one that clarifies, in particular, the source sample and provides transparency of methodology that aids in assessing the true context of the results, their appraisal and interpretation. Such uniform standards would allow better synthesis of existing evidence for healthcare decision-making, as well as highlighting gaps in knowledge and, perhaps, reducing the reinventing of the wheel.

This could be achieved through communication to clinical researchers that these aspects of study design and conduct are of critical importance. Those who review research, such as the global burden of disease exercise, currently cannot include all the research which has been published because of the limitations of design, conduct and reporting. This represents a colossal waste of effort and resources.

How do we integrate these lessons learned to make progress in neuroepidemiology? Preliminary work shows that putting qualitative and quantitative data together into a population model is possible using sophisticated modelling, but only if the data being incorporated is robust. Dementia and its subtypes remains a challenge because the evidence from the biomedical and clinical worlds does not represent closely what occurs in populations. The clinical criteria for dementia and subtypes are open to variable interpretation, leading to considerable variation in subtypes. The settings will determine the phenotype particular clinicians see. People with dementia and their caregivers will have jumped through many hoops to come to the attention of particular clinicians and the nature of these hoops (old age psychiatric services versus neurology will affect the nature of the dementia 
seen, as outlined earlier). A key development is the UK National Dementia Strategy, an initiative which has brought together patient, caregiver, clinical and psychiatric expertise and grassroots representation. It has many aims including the improvement of dementia in the general population and those whose job may not be to treat dementia (e.g. in a hospice), but who will often come in touch with people with dementia and will be important in their lives. Initiatives are underway to bring awareness of dementia and its treatments into the curriculum of nurses and medics. This isn't evidence-based medicine or management; it is largely good sense. The patients and their families are the ones asking for this support. Often, patient groups are the first to raise the concern regarding healthcare delivery in the UK. A major concern has been the long lag to getting an accurate diagnosis and treatment. Support to caregivers is another area. A new service is to be introduced which is being piloted with peer advisors and dementia advisors in different geographical locations to allow patients with dementia to cope with the healthcare system and sort out care.

However, demonstrable improvements are often anecdotal. Public health-driven evaluations need to be run alongside such initiatives, so lessons learned can be generalized and transferred to other settings, and the costs and benefits assessed. Organizational support needs to be well-planned and funded, so the information generated is clear enough for the implications to be generalizable.

One of the relatively neglected areas in dementia is the end of life. All the key issues regarding end-of-life decisions are made more acute in the presence of dementia - to resuscitate or not, to treat intercurrent infections, the role of the advance directive, end-of-life feeding. Simple aspects such as the place of death are important and it is notable that large proportions of the oldest old move across different settings in the last year of life, a factor that must affect quality of care and continuity. Dementia is highly prevalent at the end of life particularly in the oldest old. These are important areas for practical research.

Lastly, we need to add value. Questions like: is imaging (blood tests, screening tools, biomarkers, etc.) teaching or adding enough information to justify the cost in dementia diagnosis or care? Perhaps such health service research dampens the more exciting work but it still needs to be done. Massive changes are needed to improve the quality of life in elderly patients with dementia. The principles or the lessons learned should be transferable, but we need to communicate them better. Funding will vary according to regional priorities, and naturally the response will be varied due to socio-cultural differences. The evidence combined with local health economics encourages the 'responsible transfer of knowledge'.

\section{Reference}

1 STROBE statement-checklist of items that should be included in reports of observational studies (STROBE initiative). Int J Public Health 2008;53:3-4.

Editor's note: The session planned to cover EBM and epidemiological updates in Alzheimer's disease or dementia, however, the small number of attendees allowed for a broader discussion as above.

\section{Traumatic Brain Injury: Evidence- Based Management and Epidemiology Updates}

\section{Hans von Holst \\ Karolinska Institutet and Royal Institute of Technology, Department of Neurosurgery, Karolinska University Hospital, Stockholm, Sweden}

Evidence-based management strategies are rare in traumatic brain injury (TBI) cases. Clearly, one can not make informed choices without information. What is considered reliable, valid and clinically relevant? How do systematic reviews and epidemiological data support the information? Is there a way to consistently synthesize the best evidence and not simply rely on expert opinion? Varying degrees of certainty exist: Standards (high certainty but uncommon), Guidelines and Options (low certainty). Guidelines should be the most beneficial as they are revised every few years and contain up-to-date information. The major barrier to acceptance is the time it takes to collect, synthesize and produce new knowledge in a reliable, digestible format.

Nevertheless, there have been dramatic improvements in TBI management over the past 20 years. The combination of epidemiology with evidence-based medicine produces practical information and leaps forward in the prevention and awareness regarding the early indicators of TBI. Injury-related mortality varies widely globally as does the incidence of TBI. It is expected as developing countries improve their economies and infrastructure, there will be an increase in TBI and TBI-related mortality. Furthermore, it is likely that the risk factors in these countries will be proportionately different.

In general, for every TBI-related death, there are about 10 admissions and about 30 emergencies; in $70-80 \%$ of cases, the brain injury is mild. Half of the injuries arise from traffic accidents, a quarter from falls, and the rest split almost evenly between violence and leisure activities. The risk factors identified were age (first peak incidence at 18-24 years and a second at $>65$ years), gender (males were $2-3$ times more likely to have TBI than females), sociality (risk 3 times less when educated), and drugs (primarily when persons under the influence cause traffic accidents). In recent years, falls as a risk factor for TBI are increasing (particularly in $>65$ year olds) as transportation injuries are decreasing. In mild TBI, prevention strategies should take into account these age and gender differences.

Usually, there are 3 settings that could impact TBI management and outcomes. These settings include the short-term prehospital care from the scene of accident and transportation to the hospital, hospital care at the neurointensive care and the long-term rehabilitation. How does transportation to the nearest hospital or to the nearest neurology center affect outcome? What was most important at the scene of the accident was access to advanced technology and medical competence in the vehicle, regardless whether it was a van or helicopter. In Sweden, as well as in other industrial countries, the ambulance staff is well educated in first aid. Also, in some helicopter transportation, it is requested that an anesthesiologist participates.

When the case arrives at the hospital, which specialty should handle the emergency or potential TBI? Although the evidence is not definitive, the Swedish practice is that the general surgeon is 
the leader in the emergency room. The whole body needs to be considered, e.g. hypotension can occur for several reasons, not just because of brain- or nervous system-related injuries.

There are numerous illustrative cases in TBI management where it would be impossible to do a randomized controlled trial. Further challenges come with the use of imaging technology or other monitoring devices (e.g. intracranial pressure) and followup. Imaging can not distinguish whether to wait, treat, or operate. Indeed, injuries can have effects several days after the initial event. However, there is strong evidence that the mechanical pattern of injury influences the consequences to brain tissue (it is crucial to know about the accident details).

Thanks to computer technology, major advances have been made in understanding the biomechanical effects of brain injury. A better appreciation of the consequences of hematoma and destroyed nervous tissue is possible with computer simulations. The improvement of imaging technology will probably define new injury criteria, which might result in a better outcome.

Computer modeling techniques combined with MRI data and impact information can reconstruct and possibly predict changes in the brain. This data could also aid in explaining changes in the brain performance or outward personality changes over time to the patient and family, thereby, they are convinced to switch the management to a more active treatment.

Computer technology has also led to the development of a Multi-Impact Protection System, which takes into account that most impact injuries comes from an oblique angle. One helmet prototype has demonstrated it can reduce the transfer of impact energy up to $40-60 \%$ (research phase).

An evidence-based practice includes primary prevention measures and recognition of the early indicators of TBI, which allows effective management and reduction of the injury burden in the community. However, changing paradigms in treatment take a long time to prove and there are many challenges on the way. New TBI management strategies should be available soon based on computer modeling and validation of the technology.

\section{Biostatistics for Non-Biostatisticians}

\section{Derrick A. Bennett}

Clinical Trial Service Unit and Epidemiological Studies Unit (CTSU), Nuffield Department of Clinical Medicine, University of Oxford, Oxford, UK

Statistics involves problem solving by:

- Exploring the context, i.e. obtaining sufficient background information to formulate the problem carefully

- Collecting the necessary data in a valid way

- Carrying out a preliminary examination of the data

What inferences can we make about the true magnitude of the difference between 2 population means, given the observed magnitude of the difference between 2 sample means? A population is the totality of the measurements or counts obtainable from all objects possessing some common specified characteristic. A population can vary in size. We cannot usually measure or count the entire population. So we have to take a sample from the population and make inferences about the population from the sample.

A sample is a set of measurements which constitutes part of a population. We can have a random sample which is a sample in which any one measurement in the population is as likely to occur as any other. Statistics are used to extrapolate findings from a sample to a population.

Assuming the sample is representative, we use estimation to calculate a sample statistic to make our best guess about the true population parameter. The method of estimation provides a confidence interval: a statement regarding the likely magnitude of the population parameter. A bigger sample will give a narrower confidence and so more likely that sample estimate will be close to population parameter.

\section{Aim of Hypothesis Testing}

If the true population means are in fact the same, how likely are we to have observed a difference of this magnitude or more in sample means? A hypothesis is some testable belief or opinion, and hypothesis testing is the process by which the belief is tested by statistical methods. The test statistic is calculated from the observed value of the quantity of interest and the value expected if the null hypothesis were true.

For example, a sample prevalence may be compared to some hypothesized prevalence value. In many cases in medical research, the hypothesised value will be equal to zero. Therefore, the test statistic will be the ratio of the observed value to its standard error. The comparison of the test statistic with the appropriate distribution will return a $\mathrm{p}$ value, which will indicate the probability of obtaining a value as large as or more extreme than the test statistic when the null hypothesis is true. The cutoff level for statistical significance in medical research is conventionally taken as 0.05 (5\% significance level). If the $\mathrm{p}$ value is less than the stated cutoff, the null hypothesis can be rejected. For genetics, a $\mathrm{p}<5 \times 10-^{7}$ is sometimes necessary, as there are many genetic markers considered.

A Type I error is when you claim there is a difference when, in fact, there is not (alpha). A Type II error is when your data fail to detect a true difference between the groups (beta).

\section{Confounding}

Confounding is defined as the imbalance in other risk factors, measured or unmeasured, between exposed and unexposed subjects. All non-randomised studies can be affected by confounding. It is essential to ascertain the degree of confounding when interpreting the findings of an epidemiological study.

Confounding can be dealt with at the design stage (via restriction or matching) or the analysis stage. The design stage is usually preferable as this ensures that most of the known confounders have been carefully considered.

Stratification can be used which compare rates within subgroups (strata) of subjects with apparently otherwise similar risk. It is not always clear what is a confounder and what is a real effect or exposure. Differences in opinion exist, particularly when the underlying processes are not well understood. The analysis is either crude (without taking into account possible confounders) or adjusted. Adjustment is used when there are many confounders and analysis via multivariable analysis (e.g. logistic, Cox regres- 
sion) is often required. One heuristic to tell whether to adjust for a potential confounder is to see if the regression coefficient for our exposure variable changes by $10 \%$ with inclusion of the variable. It is not a good idea to keep multiple variables that are already highly correlated (e.g. body mass index, waist circumference) in the same model.

High quality of reporting of medical research is important in order to inform the members of the scientific community as well as members of non-scientific community. Statistics make it easier to justify study sample size and help find out what information is hiding in that huge array of biomedical data, i.e. 'The Truth'. Thus, one can think of the measurement as:

\section{Measurement $=$ The Truth + Bias + Random Error}

A good study design will minimize bias and large sample sizes decrease random error. When publishing, one should always critically appraise the design and offer both $\mathrm{p}$ and confidence interval values for the reader to assess the level of bias and error. 